\title{
Interference Aware Routing Protocols for Underlay Cognitive Radio Networks
}

\author{
Sami Touati *, Hatem Boujemaa ** and Nazha Abed * \\ (*) King Saud University, Kingdom of Saudi Arabia \\ (**) University of Carthage, Sup'Com, COSIM Laboratory, Tunisia \\ stouati@ksu.edu.sa, boujemaa.hatem@supcom.rnu.tn, nabed@ksu.edu.sa \\ Received: March 15, 2021. Revised: April 12, 2021. Accepted: April 15, 2021. Published: April 23, 2021.
}

\begin{abstract}
In this paper, optimal and sub-optimal routing protocols are proposed for cognitive radio networks. We first investigate optimal routing that consists in searching among all paths, the one that minimizes the end-to-end outage while verifying interference constraint to primary receiver. A sub-optimal one-hop routing is proposed where the best relay is selected in each hop. The last routing protocol consists in decomposing the network in many sub-networks composed of $K$ hops. Then the best route is determined in each of these sub-networks so that the $\mathrm{K}$ hops-outage probability is minimized. The proposed K-hops routing allows a good compromise between complexity and performance. The performance of different routing protocols are evaluated through simulation results in terms of outage probability.
\end{abstract}

Keywords : Routing protocols, Underlay cognitive radio networks, Multihop relaying, Rayleigh fading channels.

\section{INTRODUCTION}

Cognitive radio has been recently proposed as a potential enabler to use efficiently the radio spectrum. Its idea is based on the dynamic spectrum access [1]-[2] which allow unlicensed secondary users to access the spectrum opportunistically without adversely affecting the transmission performance of the license holders, called primary users ${ }^{1}$. To share the spectrum in an opportunistic manner, three approaches have been proposed namely: interweave, underlay and overlay. The interweave approach allows secondary transmissions over the spectrum bands left unused by the primary users. However, this approach requires challenging capabilities of sensing and prediction of primary activities, especially when primary users are highly dynamic. In underlay approach [3], primary and secondary transmissions are done simultaneously over the same spectrum band. The secondary users must keep the interference at the primary receiver (PR) less than a predened level. This approach allows the secondary users to access directly to the spectrum. Besides, secondary users must control adequately its transmit power in order to respect the imposed primary constraint. Moreover, the secondary receivers (SRs) suffer from the interference caused by the primary transmitter (PT). In the overlay transmission approach, secondary transmitter (ST) devotes a part of its transmit power to relay the primary data and hence improve the performances of primary transmissions in exchange of spectrum access time

\footnotetext{
${ }^{1}$ This work was supported by the Research Center of College of Computer and Information Sciences, King Saud University, under Grant No. RC1303100. The authors are grateful for this support.
}

[4]. In order to not degrade the performance of the primary network, the secondary source has to transmit with a low power which reduces the throughput of the primary network. To circumvent this problem, we can use relaying in the secondary network where some relays help the source when delivering its message. A relay can be a mobile station of the secondary network. In practical networks, there are multiple paths where the message can be routed from the source to the destination through multihops. The aim of this project is to propose different routing protocols for underlay cognitive radio networks.

Routing protocols for cognitive networks were studied in [522]. Joint routing, relay selection and spectrum allocation were investigated in [5-7]. Routing protocols has been proposed in [8-9] where the metric takes into account transmission, link access and queuing delays. However, [8-9] haven't taken into account the generated interference to primary network. The interference is an important aspect in cognitive radio networks. If the generated interference is large, secondary nodes should stop transmitting. Routing protocols using link availability were suggested in [10]. A link is considered available if the transmitter and receiver are within the transmission range and if the generated interference to primary network is low. An overview of routing protocols was provided in [11]. Two main categories were discussed. The first one assumes a full spectrum knowledge whereas the second one requires only local spectrum information. Depending on the primary user activity, static mesh routing, dynamic routing and opportunistic forwarding were proposed in [12-13] for cognitive networks. The proposed protocol is based on location and channel usage information. Interference aware routing was introduced in [14] in order to minimize interference to primary receiver while reducing transmission delays in the secondary network. Dynamic secure routing was proposed in [15] to avoid jamming attacks. The end-to-end throughput was maximized in [16] by optimizing channel selection for a given routing path. Adaptive routing has been investigated in [17] for fast varying channels. Routing protocols were proposed in [18] for cognitive radio networks to balance energy consumption and minimize routing overhead. In [19], packets are routed by taking into account outage probabilities. Spectrum aware routing was proposed in [20] for discontinuous orthogonal frequency division multiplexing-based cognitive networks. A cross-Layer routing protocol was used in [21] when the channel availability is less 
than the communication time. Routing metrics was computed using packet error probability and delays. Cognitive tree-based routing has been suggested in [22] to support many wireless networks. Cognitive aware end-to-end metric has been used to select the best route. To summarize, except [14], all previous techniques investigated route selection without taking into account the generated interference to primary network which is one of the main objectives of the project. Also, previous works tackled optimal path selection based on transmission delays or end-to-end throughput without quantifying its complexity. Our paper aims at finding some suboptimal routing protocols based on outage probabilities to have a good compromise between performance and complexity. The parameters of the proposed suboptimal routing protocol will be adjusted in order to have close performance to the optimal one. To the best of our knowledge, optimal and suboptimal interference aware routing for cognitive underlay radio networks have not been previously proposed or studied.

The paper is organized as follows. The next section presents the system model whereas section IV describes optimal and suboptimal routing protocols. Section V

\section{SYSTEM MODEL}

The system model is shown in Fig. 1. The primary network is composed of a Primary Transmitter (PT) and a Primary Receiver (PR).The secondary network is composed of a source $S$, a destination $D$ and $M L$ relays. There are $M$ possible relays in the $i$-th hop denoted by $R_{i, j}$ where $1 \leq j \leq M$. Transmission phase is composed of $L+1$ phases. In the first one, the secondary source $S$ transmits a signal to relays of the first hop $R_{1, j}$. During the second phase, a relay is selected in the set $\left\{R_{1, j}\right\}_{1 \leq j \leq M}$ using the routing protocol, to amplify the signals to the next relays $\left\{R_{2, j}\right\}_{1 \leq j \leq M}$. The same process is continued through the remaining hops. In the last $L+1$ phase, a relay is selected in the set $\left\{R_{1, j}\right\}_{1 \leq j \leq M}$ to amplify the signal to the destination.

\section{ROUTING STRATEGIES}

\section{A. Optimal routing}

Interference aware optimal routing consists in selecting the path among $M^{L}$ that minimizes the end-to-end outage probability by considering only paths corresponding to relays verifying interference constraints.

For a path $j$, the outage probability is the probability that the end-to-end AF SNR is below the threshold $\gamma_{T}$ :

$$
P_{\text {out }, j}=P\left(\gamma_{j}^{e} \leq \gamma_{T}\right),
$$

where $\gamma_{j}^{e}$ is the equivalent end-to-end SNR of the $j$-th path $\left(1 \leq j \leq M^{L}\right)$ given by [23]

$$
\gamma_{j}^{e}=\frac{1}{\sum_{i=1}^{L+1} \frac{1}{\gamma_{j, i}}},
$$

$\gamma_{j, i}$ is the SNR of the $i$-th hop for the $j$-th path. The selected path is obtained by searching the path offering the highest end-to-end SNR among the set $S$ of paths composed of relays verifying interference constraints to primary receiver PR :

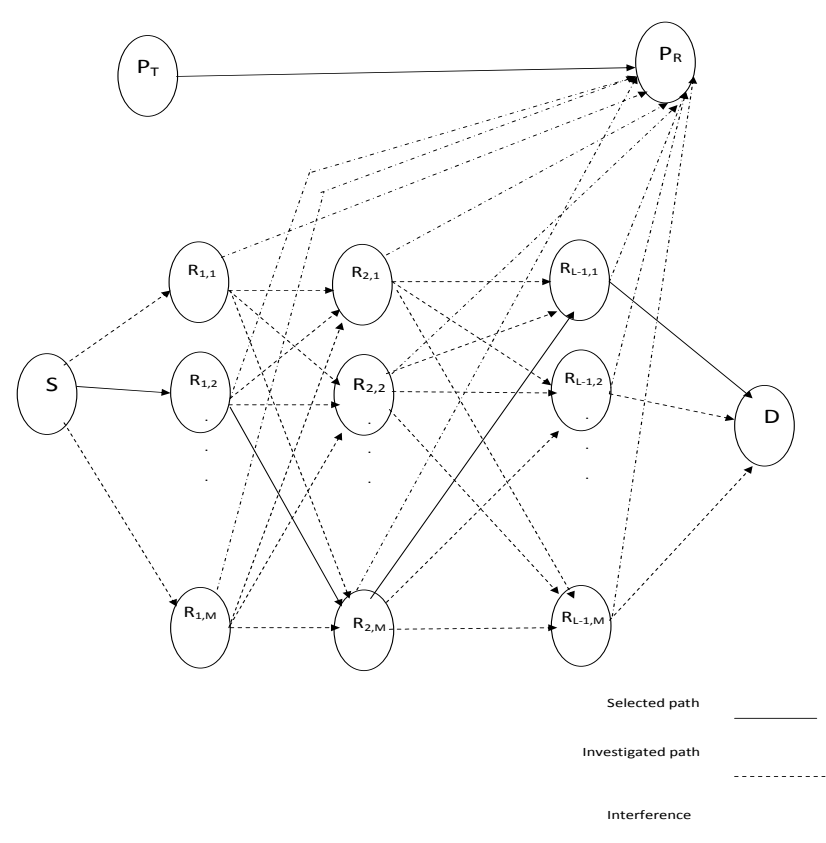

Fig. 1. System model.

$$
j s e l=\underset{j \in S}{\arg \max } \gamma_{j}^{e} .
$$

If there is no available path, which means that at a given hop there no relays verifying interference constraints, the transmission is made by the source through the direct link.

\section{B. One hop suboptimal routing}

In this routing protocol, in the $i$-th hop, we select the relay that minimizes the outage probability which is equivalent to choosing the relay with the highest SNR :

$$
R_{i, s e l}=\underset{k \in S_{i}}{\arg \max } \gamma_{R_{i-1, s e l}} R_{i, k},
$$

where $S_{i}$ is the set of relays in the $i$-th hop verifying interference constraints and $R_{0, s e l}=S$ which means that the source transmits the signal in the first hop.

\section{K-hops suboptimal routing}

$\mathrm{K}$ hops routing consists in decomposing the network in subnetworks composed of $K$ hops then the best path is selected in each subnetwork :

$$
j s e l=\underset{j \in S}{\arg \max } \gamma_{j}^{e, s u b},
$$

where $S$ is the set of paths in the subnetwork verifying the interference constraint and

$$
\gamma_{j}^{e, s u b}=\frac{1}{\sum_{i=1}^{K} \frac{1}{\gamma_{j, i}}},
$$


$\left\{\gamma_{j, i}\right\}_{i=1, \ldots, K}$ are the SNRs caracterizing the $K$ hops of the $j$-th path in the subnetwork.

\section{PERFORMANCE ANALYSIS OF OPTIMAL ROUTING} by

The end-to-end SNR of the $j$-th path can be upper bounded

$$
\gamma_{j}^{e}<\gamma_{j, \min }^{e}=\min _{1 \leq i \leq L+1}\left(\gamma_{j, i}\right)
$$

This upper bound is tight and has been used in many studies [cite]. For Rayleigh fading channels, $\gamma_{j, \min }^{e}$ follows an exponential distribution with mean [23]

$$
\overline{\gamma_{j, \min }^{e}}=\frac{1}{\sum_{i=1}^{K} \frac{1}{\overline{\gamma_{j, i}}}} .
$$

The outage probability of the $j$-th path is given by

$$
P_{\text {out }, j}=P\left(\gamma_{j}^{e} \leq \gamma_{T}\right)>P\left(\gamma_{j, \min }^{e} \leq \gamma_{T}\right)=1-e^{-\frac{\gamma_{T}}{\gamma_{j, \min }^{e}}} .
$$

The SNR of the selected path according to optimal routing is given by

$$
\gamma_{j s e l}^{e}=\max _{j \in S} \gamma_{j}^{e} .
$$

Therefore, the end-to-end outage probability of the selected path is given by

$$
P\left(\gamma_{j \text { sel }}^{e} \leq \gamma_{T}\right)=\prod_{j \in S} P\left(\gamma_{j}^{e} \leq \gamma_{T}\right)>\prod_{j \in S}\left[1-e^{-\frac{\gamma_{T}}{\gamma_{j, \mathrm{~min}}^{e}}}\right]
$$

The previous equation gives the outage probability for a given set $S$ of paths verifying interference constraints to primary receiver. A lower bound of the average outage probability is given by

$$
\begin{aligned}
P_{\text {out }}> & \sum_{s \neq \emptyset} P(S=s) \prod_{j \in S}\left[1-e^{-\frac{\gamma_{T}}{\gamma_{j, \text { min }}^{e}}}\right], \\
& +P(S=\emptyset)\left[1-e^{-\frac{\gamma_{T}}{\gamma_{S D}}}\right]
\end{aligned}
$$

where $P(S=s)$ is the probability that relays in set $s$ verify the interference constraint and the remaining ones generate interference larger than a predefined threshold $T$ :

$$
P(S=s)=\prod_{R_{l, k} \in s} P\left(I_{R_{l, k} P_{R}}<T\right) \prod_{R_{m, p} \notin s} P\left(I_{R_{m, p} P_{R}}>T\right),
$$

where $I_{R_{l, k} P_{R}}$ is the generated interference at primary receiver $P_{R}$ from relay $R_{l, k}$.

For Rayleigh fading channels, $I_{R_{l, k} P_{R}}$ is exponentially distributed and we have

$$
P\left(I_{R_{l, k} P_{R}}<T\right)=1-e^{-\frac{T}{\bar{I}_{R_{l, k} P_{R}}}} .
$$

When there is no available path, i.e. $s=\emptyset$, the transmission is made by the source and the outage probability corresponds to that of the direct link.

\section{NUMERICAL AND SIMULATION RESULTS}

In this section, we present some theoretical and simulation results of optimal and suboptimal interference aware routing in terms of SNR outage probability. We have considered a network composed of $L+1=5$ hops and $M=4$ possible relays in each hop. The distance between any relay and the primary receiver is $d_{R_{l, k} P_{R}}=1$. The distance between the secondary source and the destination $d_{S D}=5$. The distance between the source and the relays of the first hop is equal to $d_{S R_{1, j}}=1$. The same distance is maintained for the remaining hops $d_{R_{1, j} R_{2, j}}=d_{R_{2, j} R_{3, j}}=d_{R_{3, j} R_{4, j}}=d_{R_{4, j} D}=1$.

Fig. 2 shows the outage probability of optimal routing that consists in searching all $M^{L}$ paths as described in section III.A. The SNR threshold was varied from $\gamma_{T}=0,3$ and $5 \mathrm{~dB}$. The interference threshold was set to $T=0 \mathrm{~dB}$. We observe that the simulation results are in a good agreement with the theoretical curves. We also observe that the outage probability increases as $\gamma_{T}$ increases.

Fig. 3 compares the outage probability of optimal routing, one hop routing and $K=2$ hops suboptimal routing for $\gamma_{T}=0 \mathrm{~dB}$. The last suboptimal routing consists in dividing the networks in two subnetworks. The first subnetwork is composed of the first two hops. The second one contains the remaining hops. Then, we use the best path in each subnetwork. We observe that optimal routing offers the best performance. $K$ hops routing offers better performance than one-hop routing and can be considered a good solution to have a good compromise between complexity and performance.

Fig. 4 shows the outage probability of optimal routing for different values of the interference threshold $T=+\infty, 1,0.5$ and 0.4 and $\gamma_{T}=5 \mathrm{~dB}$. We observe that the outage probability increases as $T$ decreases since there will be less available relays and paths.

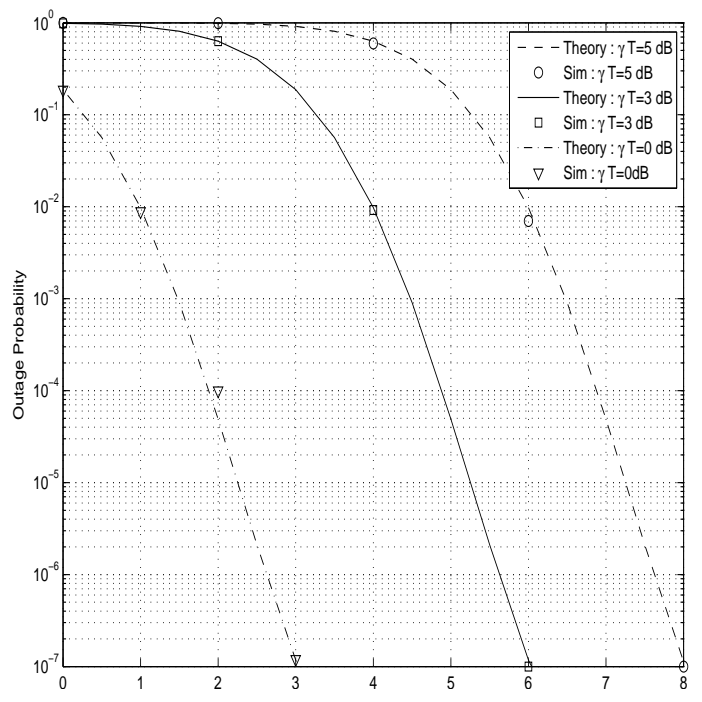

Fig. 2. Outage probability of optimal routing for $\gamma_{T}=0,3$ and $5 \mathrm{~dB}$. 


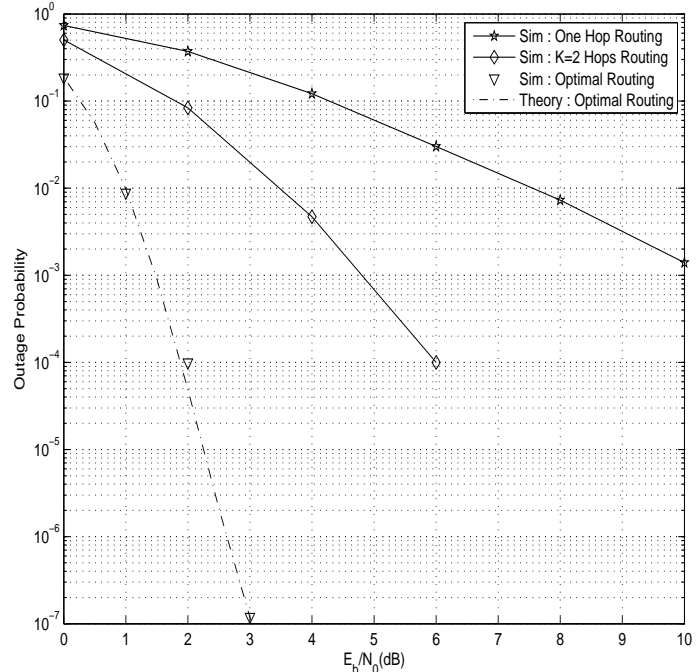

Fig. 3. Outage probability of optimal and suboptimal routing for $\gamma_{T}=0 \mathrm{~dB}$, $T=0 d B, M=4, L=4$

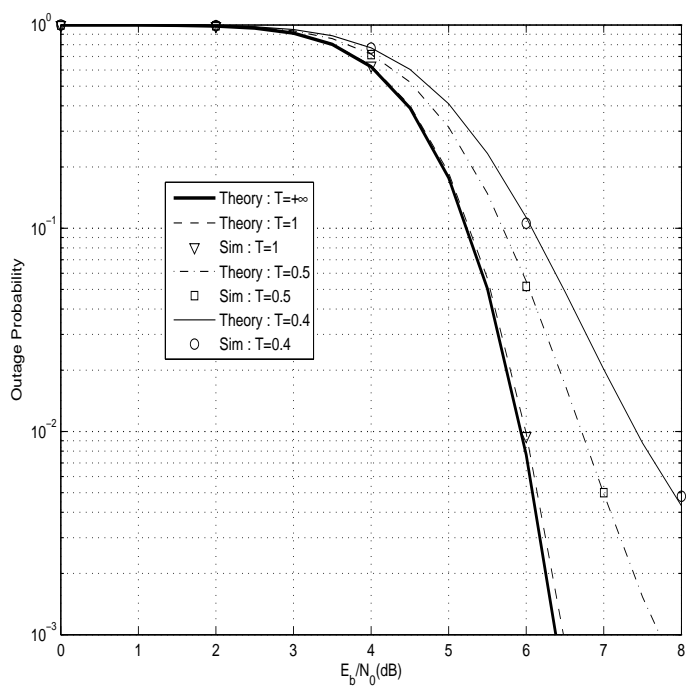

Fig. 4. Outage probability of optimal routing for different values of the interference threshold $T$.

\section{CONCLUSIONS AND PERSPECTIVES}

In this paper, we have proposed interference aware optimal and suboptimal routing protocols for underlay cognitive radio networks. Optimal routing consists in searching the best path among all available paths. Available paths are composed of relays verifying interference constraints to primary receiver. One hop suboptimal routing consists in selecting the relay with the largest SNR at each hop. K hops suboptimal routing consists in decomposing the network on subnetworks composed of $K$ hops then the best path is selected in each subnetwork. We have shown that optimal routing offers better performance than suboptimal routing. $K$ hops routing offers better performance than one-hop routing. It offers a good compromise between complexity and performance. As a perspective, we will evaluate the theoretical performance of the suboptimal routing protocols.

\section{REFERENCES}

[1] I. Mitola, J. and J. Maguire, G.Q., Cognitive radio: making software radios more personal, IEEE Personal Commun., vol. 6,pp. 1318, Aug. 1999.

[2] I. F. Akyildiz, W.-Y. Lee, M. C. Vuran, and S. Mohanty, Next generation/dynamic spectrum access/cognitive radio wireless networks: A survey, Computer Networks, vol. 50, no. 13, pp. 21272159, 2006.

[3] S. Haykin, Cognitive radio: brain-empowered wireless communications, IEEE J. Sel. Areas Commun., vol. 23, no. 2, pp. 201220, Feb. 2005

[4] R. Menon, R. Buehrer, and J. Reed, Outage probability based comparison of underlay and overlay spectrum sharing techniques, IEEE Int. Symp. on New Frontiers in Dynamic Spectrum Access Networks, 2005.

[5] L. Ding, T. Melodia, S. N. Batalama, J. D. Matyjas, "Distributed routing, relay selection and spectrum allocation in cognitive and cooperative Ad-Hoc Networks", IEEE Conference on Sensor Mesh and Ad-hoc Communications and netwotks, 21-25 June 2010.

[6] D. Lei, M. Tommaso, B. Stella, J.D. Matyjas, M. Medley, "Cross-Layer routing and dynamic spectrum allocation in cognitive radio Ad Hoc Networks", IEEE Transactions on Vehicular Technology, Vol. 59, Issue 4, pp. 1969-1979, 2010.

[7] K. R. Chawdhury, I. Akyildiz, "CRP : A routing protocol for cognitive radio Ad-Hoc Networks", IEEE Journal on Selected Areas in Communications, Vol. 29, Issue 4, pp. 794-804, 2011.

[8] S. C. Lin, K. C. Chen, "Spectrum Aware opportunistic routing in cognitive radio networks", IEEE GLOBECOM, 2010.

[9] M. Caleffi, I. Akyildiz, P. Luigi, ”OPERA : optimal routing metric for cognitive radio Ad-Hoc Networks",Vol. 11, Issue. 8, pp. 2884-2894, IEEE Trans. on Wireless Communications, 2012.

[10] Q. Guan, F. R. Yu, S. Jiang, G. Wei, "Prediction-based topology control and routing in cognitive radio mobile Ad Hoc Networks", IEEE Trans. on Veh. Technology, vol. 59, No. 9, Nov. 2010.

[11] M. Cesana, F. Cuomo, E. Ekici, "Routing in cognitive radio networks : challenges and solutions", Elsevier Ad Hoc Networks, pp.1-21, 2010.

[12] H. Khalife, N. Malouch, S. Fdida, "Multihop cognitive radio networks : to route or not to route", IEEE Network, pp.1-11, 2009.

[13] L. Yongkang, C. Lin, S. X. Sherman, "Spectrum-aware opportunistic routing in multi-hop cognitive radio networks", IEEE Journal on Selected Areas in Communications, Vol. 30, Issue 10, pp. 1958-1968, 2012.

[14] Z. Quanyan, Y. Zhou, S. J. Bin, H. Zhu, B. Tamer, "Interference aware routing game for cognitive radio multi-hop networks", IEEE Journal on Selected Areas in Commun., vol. 30, issue 10, pp.2006-2015, 2012.

[15] Z. Quanyan, S. Ju Bin, B. Tamer, "Dynamic secure routing game in distributed cognitive radio networks", GLOBECOM, pp.1-6, 2011.

[16] M. Brendan, T. Jian, J. Ivan, S. David, "On routing and channel selection in cognitive radio mesh networks", IEEE Transactions on Vehicular Technology, Vol. 61, Issue 9, pp. 4118-4128, 2012.

[17] H. X. Lin, W. Gang, H. Fei, S. Sunil, "Stability-Capacity-Adaptive routing for high mobility multihop cognitive radio networks", IEEE Trans. on Vehicular Technology, vol. 60, issue 6, pp. 2714-2729, 2011.

[18] S. M. Kamruzzaman, E. Kim, D. G. Jeong, W. S. Jeon, "Energy aware routing protocol for cognitive radio ad hoc networks," Vol. 6, Issue 14, pp. 2159-2168, IET Communications, 2012.

[19] E. Dall Anese, G. B. Giannakis, "Statistical routing for multihop wireless cognitive networks", IEEE Transactions on Selected Areas in Communications, Vol. 30, Issue 10, pp. 1983-1993, 2012.

[20] Y. S. Chen, S. H. Liao, "Spectrum aware routing in discontinuous orthogonal frequency division multiplexing based cognitive radio ad hoc networks", IET Networks, Vol. 1, Issue 1, pp. 20-33, 2012.

[21] H. K. Cheng, M. Maode, Q. Yang, "An opportunistic service differentiation routing protocol for cognitive radio networks", IEEE GLOBECOM pp. 1-5, 2010.

[22] Z. Bin, T. Yasuhisa, H. Akio; Y. Akira, O. Sadao, "Tree-based routing protocol for cognitive wireless Access networks", IEEE WCNC conference, pp. 4204-4208, 2007.

[23] M. O. Hasna, M. S. Alouini, "End-to-end performance of transmission systems with relays over rayleigh fading channels", IEEE Trans. Wireless Commun., Vol. 2, pp. 1126-1131, Nov. 2003.

\section{Creative Commons Attribution License 4.0 (Attribution 4.0 International, CC BY 4.0)}

This article is published under the terms of the Creative Commons Attribution License 4.0 https://creativecommons.org/licenses/by/4.0/deed.en_US 